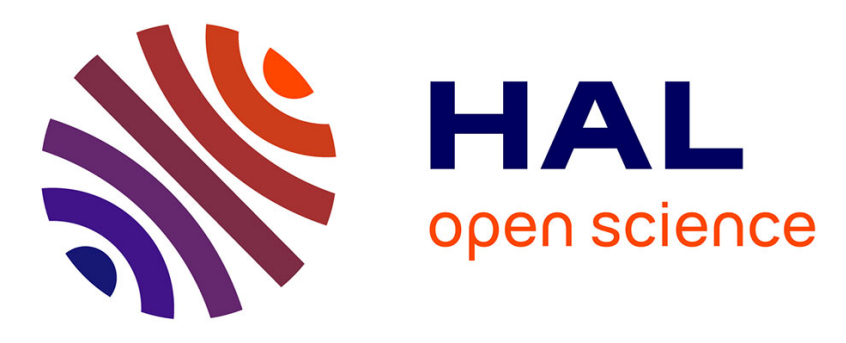

\title{
Path selection rules for droplet trains in single-lane microfluidic networks.
}

Axelle Amon, Alexandre Schmit, Louis Salkin, Laurent Courbin, Pascal Panizza

\section{- To cite this version:}

Axelle Amon, Alexandre Schmit, Louis Salkin, Laurent Courbin, Pascal Panizza. Path selection rules for droplet trains in single-lane microfluidic networks.. Physical Review E : Statistical, Nonlinear, and Soft Matter Physics, 2013, 88 (1), pp.013012. 10.1103/PhysRevE.88.013012 . hal-00854171

\section{HAL Id: hal-00854171 https://hal.science/hal-00854171}

Submitted on 26 Sep 2013

HAL is a multi-disciplinary open access archive for the deposit and dissemination of scientific research documents, whether they are published or not. The documents may come from teaching and research institutions in France or abroad, or from public or private research centers.
L'archive ouverte pluridisciplinaire HAL, est destinée au dépôt et à la diffusion de documents scientifiques de niveau recherche, publiés ou non, émanant des établissements d'enseignement et de recherche français ou étrangers, des laboratoires publics ou privés. 


\title{
Path selection rules for droplet trains in single-lane microfluidic networks
}

\author{
A. Amon, A. Schmit, L. Salkin, L. Courbin, and P. Panizza \\ IPR, UMR CNRS 6251, Campus Beaulieu, Université Rennes 1, 35042 Rennes, France
}

(Dated: September 12, 2013)

\begin{abstract}
We investigate the transport of periodic trains of droplets through microfluidic networks having one inlet, one outlet, and nodes consisting of $\mathrm{T}$ junctions. Variations of the dilution of the trains, i.e. the distance between drops, reveal the existence of various hydrodynamic regimes characterized by the number of preferential paths taken by the drops. As the dilution increases, this number continuously decreases until only one path remains explored. Building on a continuous approach used to treat droplet traffic through a single asymmetric loop, we determine selection rules for the path taken by the drops and we predict the variations of the fraction of droplets taking these paths with the parameters at play including the dilution. Our results show that, as dilution decreases, the paths are selected according to the ascending order of their hydrodynamic resistance in the absence of droplets. The dynamics of these systems controlled by time-delayed feedback is complex: we observe a succession of periodic regimes separated by a wealth of bifurcations as the dilution is varied. In contrast to droplet traffic in single asymmetric loops, the dynamical behavior in networks of loops is sensitive to initial conditions because of extra degrees of freedom.
\end{abstract}

PACS numbers: 47.60.Dx 47.55.D- 47.20.Ky

\section{INTRODUCTION}

A laminar, steady, and Newtonian flow in a rigid pipe is described by the Hagen-Poiseuille equation which states $\Delta P=R Q$, with $Q$ the volumetric flow rate, $\Delta P$ the pressure drop between the pipe's inlet and outlet, and $R$ a constant having units $\mathrm{Pa} \mathrm{s} \mathrm{m}{ }^{-3}$ whose value solely depends on the fluid viscosity and the geometry of the pipe [1]. Because of the analogy between this equation and the classical Ohm's law for the analysis of electric circuits, relationships analog to Kirchhoff's laws for the electrical current and voltage drop can be written for $Q$ and $\Delta P$, respectively [1-3]. Hence, obeying to the combination rules of resistors, $R$ is known in the literature as the hydrodynamic resistance of the pipe [4].

Now, considering a fluidic network made of numerous interconnected branches, the determination of the flow rates in each branch can be difficult. For monophasic Newtonian flows, this problem has a unique solution easily derived using the analogy between fluidic networks and electric circuits mentioned above. For two-phase flows however, e.g flows in (droplet-based) digital microfluidic applications [5, 6], strong nonlinearities arise and the problem becomes challenging. Indeed, the transport of discrete elements or information through networks, such as droplet traffic in single-lane microchannels having lateral dimensions comparable to the drop size, road traffic [7], blood microcirculation [8], and cell signaling [9], are all regulated by time-delayed feedback and nonlinear couplings. When reaching a node, a droplet generally flows in the channel having the largest instantaneous volumetric flow rate $[10,11]$. Since the hydrodynamic resistance of a channel depends on the presence of flowing drops [12], the path selection of a drop at a node is monitored by the entrance and exit of the pre- ceding drops in all the branches of the network. Even in the simple case of two bifurcating channels, the traffic of drops or bubbles can be amazingly complex and yields a rich variety of dynamics including periodic and multistable traffic patterns $[11,13-16]$. However, so far investigations mostly focus on trains of bubbles or droplets flowing through a single loop [11, 13-22], while a few deal with more complex geometries [23, 24].

Here, we investigate droplet traffic in complex singlelane networks to determine whether the approaches and simple rules employed to rationalize experimental findings for a single loop are still valid. The paper is organized as follows. In section II, we briefly present the phenomena observed when studying droplet traffic in the most studied configuration, an asymmetric loop [10, 11, 13-18] and we give the basic elements used in the continuous $[10,11,15-18]$ and the discrete $[13,14]$ models that rationalize observations. In addition, we provide the complete derivations of the analytical results obtained using discrete models [13]. In section III, building on these theoretical grounds, we study droplet traffic through networks having one inlet and one outlet that can be connected using more than two different ways; the nodes of the networks are $\mathrm{T}$ junctions. We model the flow and we validate the resulting predictions with microfluidic experiments.

\section{TRAFFIC THROUGH A SINGLE-LANE ASYMMETRIC LOOP}

\section{A. Background}

The basic elements necessary to describe the transport of droplets in single-lane microfluidic conducts having lateral dimensions of the order of the drop size and 
through an asymmetric loop are the following [10, 11, 13$18,25]$ :

(a) The hydrodynamic resistance $R$ of a pipe of length $L$ and constant cross-section $S$ varies linearly with the number $N$ of drops it contains, $R=\alpha\left(L+N L_{d}\right)$. In this expression, $\alpha$ has units $\mathrm{Pa} \mathrm{s} \mathrm{m}^{-4}$ and is a function of the viscosity of the continuous phase and of the geometry of the channel cross-section [1], and $L_{d}$ is a parameter having the dimension of a length which corresponds to the effective resistive length each droplet adds to the pipe in terms of hydrodynamic resistance.

(b) The velocity $V$ of droplets flowing in such a pipe varies as $V=\beta \frac{Q}{S}$ where $Q$ is the total flow rate and $\beta$ is a dimensionless number characterizing the mobility of the drops in the pipe.

(c) At a $\mathrm{T}$ junction, a droplet always flows in the pipe having the larger total flow rate.

The validity of the expressions for $R$ and $V$ in points (a) and (b) has been demonstrated by both millifluidic and microfluidic experiments in channels having circular and rectangular cross-sections, respectively [10, 12, 18]. Such equations can be derived using a phenomenological model considering that the droplets are sufficiently far apart so that they do not interact hydrodynamically [12]. The value of $L_{d}$ depends on the geometry of the crosssection, the viscosity ratio between dispersed and continuous phases, and the droplet confinement $\rho$ defined as the ratio between the drop size and the lateral dimension of the channel [12]. The modeling of the flow described above is valid for confinements typically varying in the range $0.7-1$ for which $L_{d}$ increases with $\rho$. For smaller $\rho$, the hydrodynamic resistance appears to be nearly independent of the presence of droplets, $L_{d} \simeq 0$. For larger values of $\rho$, nonlinearities may arise because of capillary effects [11]. The mobility $\beta$ is a decreasing function of $\rho$ which varies between 2 and 1 for circular cross-sections. $\beta$ can be either larger or smaller than 1 in the case of rectangular cross-sections $[12,26]$ because of corner flows that only exist in this geometry. The path selection rule at a node given in point (c) has a limited range of validity since experiments have shown that collisions between successive drops can occur at a node and regulate traffic when droplets are close enough $[21,25]$. In what follows, we only consider situations for which the distance $\lambda$ between drops reaching a junction is sufficiently large to avoid such collisions to occur.

So far, most studies have considered one-dimensional (1-D) trains of monodisperse droplets produced at a constant rate $f$ and flowing through a single asymmetric loop (Fig. 1). The reason for considering periodic 1-D trains is threefold. First, their production is easy using robust geometry-based methods such as $\mathrm{T}$ junctions [27, 28] or Flow Focusing Devices [29]. Second, most digital microfluidic high-throughput applications in chemistry [30],

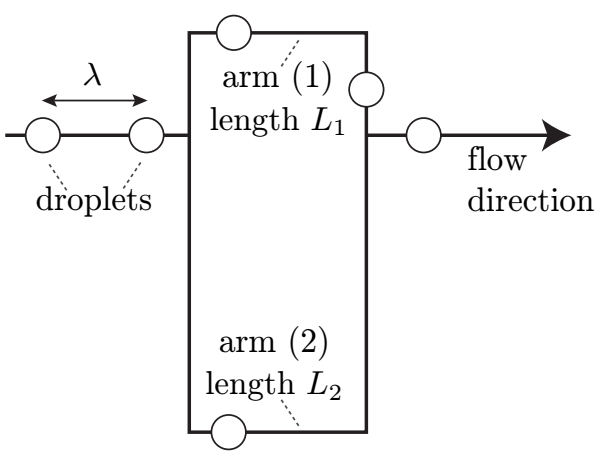

FIG. 1. Schematic of the flow model for a single-lane asymmetric loop defining $\lambda, L_{1}$, and $L_{2}>L_{1}$.

biotechnology [31, 32], and material sciences [33] require to index the drops and/or to use a space-to-time conversion, two characteristics that are inherent to 1-D trains. Finally, the use of such trains makes the modeling easier. We consider that both arms of the loop have the same constant cross-section $S$ but different lengths $L_{1}$ and $L_{2}>L_{1}$ (Fig. 1). The other parameters at play are $\beta, L_{d}$, and $\lambda$, the velocity of the droplets being $V=\lambda f$. More details on microfluidic and millifluidic set-ups able to monitor independently $L_{d}, f$, and $\lambda$ can be found in $[10,12]$. Note, however, that $L_{d}$ and $\beta$, which depend on the droplet confinement, can never be set independently.

The problem can be addressed numerically using a simple algorithm $[11,12,17,20,34]$. At each time step $\tau=\frac{1}{f}$ a droplet whose physical volume is neglected reaches the inlet node of the loop. One computes the numbers of droplets $N_{i}$ present in each arm $(i)(i=1$ or 2$)$ and the hydrodynamic resistance of this arm $R_{i}$. The drop at the inlet node is then injected in the arm having the smaller hydrodynamic resistance. Afterwards, $N_{i}$ and $R_{i}$ are updated. In each arm $(i)$, the velocity of the drops $V_{i}$ is computed using the conservation of the total flow rate and the relationships describing the transport of droplets in a pipe of constant cross-section. The droplets present in each arm $(i)$ are then moved until $N_{i}$ changes, i.e. until the exit of a drop or the entrance of a new drop. Whenever a droplet exits the system, the values of $N_{i}$, $R_{i}$, and $V_{i}$ are updated so that the displacements of all droplets are reevaluated. For a given set of parameters $\left(L_{d}, L_{1}, L_{2}\right)$, when $\lambda$ is varied, experimental and numerical findings share the following features:

- At high dilutions, all droplets flow in the shorter arm. For smaller $\lambda$, partitioning of the drops between both arms is observed. The transition between these two regimes, respectively known as the Filter and Repartition regimes, occurs at $\lambda=\lambda_{f}$ (see Fig. 2). In the latter regime, periodic patterns of droplets partitioning are obtained.

- When droplets take both paths at the junction, the total flow rates $Q_{1}$ and $Q_{2}$ in the arms, hence $V_{1}$ 
and $V_{2}$, fluctuate in time around equal mean values.

- In the Repartition regime, at a given $\lambda$, a periodic pattern is characterized by three invariants that are independent of the initial conditions, i.e. the number and the positions of the droplets initially present in the loop: the cycle time $T_{c y c}$ of the pattern (i.e. the number of drops per cycle), the fraction of drops $F_{2}$ flowing in the longer arm, and the number of "packs" $N_{\text {pack }}$ per cycle; a pack is the number of drops flowing in the shorter arm between two successive drops taking the longer arm.

- When plotted versus $\lambda$, each invariant presents series of "plateaus" separated by discontinuous transitions (see Fig. 3 through Fig. 5).

The results presented in Fig. 2 through Fig. 5 are outcomes of numerical simulations and predictions of the continuous and discrete models that are discussed in details in section II B and section II C, respectively.

\section{B. The continuous model}

To rationalize some of these findings and derive analytical expressions for $F_{2}$ and $\lambda_{f}$, one can use a continuous approach [12]. This description neglects the temporal fluctuations of the number of drops present in each arm and postulates, in the Repartition regime, the equality of the total flow rates in both arms $[10,12,14,16]$. Hence, the rate $f_{i}$ of the passing drops in the arm $(i)$ and the distance $\lambda_{i}$ between two successive drops are assumed to be constant. In the Repartition regime, using the conservations of the total flow rate and dispersed phase and writing the two relationships given in section II A [points (a) and (b)] for each arm, one obtains an analytically solvable system of four equations satisfied by $\lambda_{i}$ and $f_{i}$ (see [12] for details). With the resulting expressions for $\lambda_{i}$ and $f_{i}$, one finds that $F_{2}=\frac{f_{2}}{f}$ linearly decreases with $\lambda:$

$$
F_{2}=\frac{1}{\Lambda_{2}+1}\left(1-\lambda \frac{\Lambda_{2}-1}{2 L_{d}}\right)
$$

where $\Lambda_{2}=\frac{L_{2}}{L_{1}}$. Hence, $F_{2}\left(\lambda=\tilde{\lambda_{f}}\right)=0$ gives:

$$
\tilde{\lambda_{f}}=\frac{2 L_{d}}{\Lambda_{2}-1} .
$$

This prediction for the transition between Filter and Repartition regimes correlates with numerical results $\left(\tilde{\lambda_{f}} \sim \lambda_{f}\right.$ in Fig. 2). Expressions for the mean number of droplets $\left.<N_{i}\right\rangle=L_{i} / \lambda_{i}$ in each arm $(i)$ can also be found.

The fluctuations of $R_{i}$, which are due to the exit and entrance of droplets in the loop, remain small compared to the resistances' mean values when working with long enough arms. In that case, the model concurs well with numerical results as shown in Fig. $2[12,14,16]$. However, the model does not explain the plateaus observed

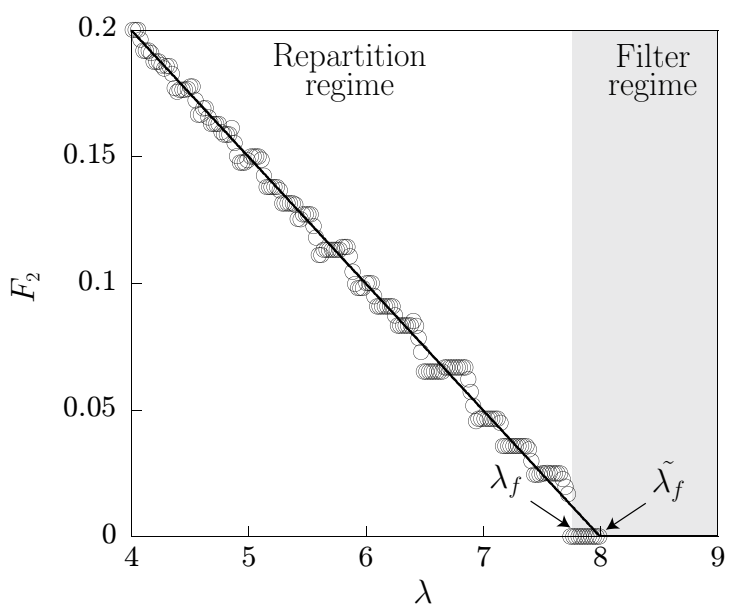

FIG. 2. Numerical fraction of droplets $F_{2}$ versus $\lambda$ for $L_{1}=100$, $L_{2}=150$, and $L_{d}=2$. The lengths have arbitrary units. The solid line is a prediction calculated using Eq. (1a).

numerically in the variations of $F_{2}$ with $\lambda$ (see Fig. 2). This limitation results from the discrete nature of the drops which a continuous approach cannot account for. Another limitation of this approach resides in the impossibility of describing the dynamics of the system.

\section{The discrete model}

\section{Introduction}

To account for the discrete nature of the droplets, one can introduce an approximation for $V_{i}$ making the problem tractable and allowing for a complete theoretical description of the dynamics $[13,14]$. This approach neglects the temporal fluctuations of the hydrodynamic resistance of the $\operatorname{arm}(i), R_{i}=\alpha\left(L_{i}+L_{d} N_{i}\right)$. One assumes that the temporal variation of $N_{i}$ does not significantly affect $V_{i}$. This "mean-field" approach is reasonable when the mean number of drops present in each arm is large or when the lengths of both arms are large compared to $L_{d}$. As shown in section II C 2, neglecting the nonlinear couplings between $N_{i}$ and $Q_{i}$ one can demonstrate that $Q_{i}$ is nearly constant and equal to one half of the incoming total flow rate in the Repartition regime so that $V_{i} \sim V / 2[13,14]$.

The time is discretized in $\tau$ units within this ideal model. Each time step, a new droplet reaches the inlet node of the loop and is injected in one of its arm. At any time step, the positions of the drops present in each arm are multiple of $\frac{\lambda}{2}$, the origin of space being located at the inlet node. Because of the finite number of possible configurations and the deterministic nature of the model, only periodic patterns are expected. A drop located in the arm $(i)$ at a position $k \frac{\lambda}{2}$ from the origin $(k \in \mathbb{N})$ has therefore reached the inlet node and selected this arm a time $k$ earlier in $\tau$ units. Since the 
droplets make a binary "choice" at the inlet, if a droplet is located at $k \frac{\lambda}{2}$ from the origin in the arm (2), a "hole" (that is, the absence of a droplet) is necessarily located at the same distance from the origin in the arm (1) and vice versa. Hence, the maximum number of droplets that can be accommodated in each arm is $T_{i}=\operatorname{ceil}\left(\frac{2 L_{i}}{\lambda}\right)$. Using the space-to-time conversion, $T_{i}$ which corresponds to the number of drops that have reached the inlet node between the entrance and exit of a given drop in the arm $(i)$, is the discrete time in $\tau$ units elapsed between these two events. In what follows, $\tau$ is the unit of time (i.e. $\tau=1$ ) and we use the superscripts ${ }^{-}$and + to respectively denote the functions evaluated just before or after injecting a new drop in the loop. The values of $N_{1}$ and $N_{2}$ just before and after the entrance of the $n$-th droplet in the loop respectively read:

$$
\begin{aligned}
N_{1}^{-}(n) & =\sum_{k=n-T_{1}+1}^{n-1} H\left[\delta^{-}(k)\right], \\
N_{2}^{-}(n) & =\sum_{k=n-T_{2}+1}^{n-1} H\left[-\delta^{-}(k)\right], \\
& =T_{2}-1-\sum_{n-T_{2}+1}^{n-1} H\left[\delta^{-}(k)\right],
\end{aligned}
$$

and:

$$
\begin{aligned}
N_{1}^{+}(n) & =\sum_{k=n-T_{1}+1}^{n} H\left[\delta^{-}(k)\right], \\
N_{2}^{+}(n) & =\sum_{k=n-T_{2}+1}^{n} H\left[-\delta^{-}(k)\right], \\
& =T_{2}-\sum_{n-T_{2}+1}^{n} H\left[\delta^{-}(k)\right] .
\end{aligned}
$$

In these expressions, $\delta(t)=\frac{L_{2}-L_{1}}{L_{d}}+N_{2}(t)-N_{1}(t)$ is the normalized difference between the hydrodynamic resistances of the two arms and $H$ is the Heaviside function. We next replace $N_{i}^{+}$by $N_{i}$ for readability.

Using the previous relationships, one can derive two equations. A balance on $N_{1}$ and $N_{2}$ over $\tau$ between the entrances of the $n$-th drop and the $(n+1)$-th one gives the first equation, that is, a recursive relationship:

$$
\begin{aligned}
\delta^{-}(n+1)=\delta^{-}(n)-2 H\left[\delta^{-}(n)\right] & +H\left[\delta^{-}\left(n+1-T_{1}\right)\right] \\
& +H\left[\delta^{-}\left(n+1-T_{2}\right)\right]
\end{aligned}
$$

During $\tau, H\left[\delta^{-}\left(n+1-T_{1}\right)\right]$ and $1-H\left[\delta^{-}\left(n+1-T_{2}\right)\right]$ drops respectively exit the loop via arms (1) and (2), whereas $H\left[\delta^{-}(n)\right]$ and $1-H\left[\delta^{-}(n)\right]$ drops enter these two respective arms. Initial conditions over a time interval are necessary to integrate the recursive relationship and to describe the dynamics of the system; this feature is common to any time-delayed systems. In our study, the positions of the drops initially present in the loop define such initial conditions. We next consider that the loop does not initially contain any drop.

Using the expression of the number of holes in the $\operatorname{arm}(1), N_{1}^{H}(n)=T_{1}-N_{1}(n)=\sum_{k=n-T_{1}+1}^{n} H\left[-\delta^{-}(k)\right]$, it is possible to show that:

$$
\begin{aligned}
\sum_{j=1}^{T_{1}} N_{2}(j) & =\sum_{j=1}^{T_{1}} \sum_{k=j-T_{2}+1}^{j} H\left[-\delta^{-}(k)\right], \\
& =\sum_{j=1}^{T_{2}} \sum_{k=j-T_{2}+1}^{j+T_{1}-T_{2}} H\left[-\delta^{-}(k)\right], \\
& =\sum_{j=1}^{T_{2}} N_{1}^{H}\left(j+T_{1}-T_{2}\right), \\
& =\sum_{j=1+T_{1}-T_{2}}^{T_{1}} N_{1}^{H}(j) .
\end{aligned}
$$

It is worthwhile noticing that this relationship is valid for any $n$ and can be written in the following form:

$$
\sum_{j=1-T_{1}+T_{2}}^{T_{2}} N_{2}(j+n)=\sum_{j=1}^{T_{2}} N_{1}^{H}(j+n) .
$$

\section{Repartition regime: The flow rate's equipartition rule}

In the Repartition regime, as observed in both numerical simulations and experiments $[10,12,14,16]$, the system naturally tends to balance the hydrodynamic resistances of both arms. As shown below a stability analysis permits to understand this phenomenon. When $\delta^{-}(n)>0$, one easily shows using Eq. (2) that $\delta^{-}(n+1)=$ $\delta^{-}(n)-2+H\left[\delta^{-}\left(n+1-T_{1}\right)\right]+H\left[\delta^{-}\left(n+1-T_{2}\right)\right]$. Hence, $\delta^{-}(n)-2 \leq \delta^{-}(n+1) \leq \delta^{-}(n)$, so that $\delta^{-}$either decreases or remains constant. Conversely, when $\delta^{-}(n)<0$, one shows that $\delta^{-}(n+1)=\delta^{-}(n)+H\left[\delta^{-}\left(n+1-T_{1}\right)\right]+$ $H\left[\delta^{-}\left(n+1-T_{2}\right)\right]$. Thus, $\delta^{-}(n) \leq \delta^{-}(n+1) \leq \delta^{-}(n)+2$, i.e. $\delta^{-}$increases or remains constant.

We now aim to show that $\delta^{+}$can only take two values after a transient regime. Distinguishing the values taken by $\delta$ before and after the entrance of the $n$-th droplet in the loop, respectively denoted $\delta^{-}(n)$ and $\delta^{+}(n)$, we use the following relations characterizing (a) the entrance of a new drop in the loop and (b) the exits of drops from the loop:

(a) $\quad . \delta^{+}(n)=\delta^{-}(n)-1$ when $\delta^{-}(n)>0$. . $\delta^{+}(n)=\delta^{-}(n)+1$ when $\delta^{-}(n)<0$.

(b) $\quad . \delta^{-}(n+1)=\delta^{+}(n)$ when none of the drops exit or two drops exit, each one passing through each arm. Such cases are possible since the residence times of the drops in the loop are different.

. $\delta^{-}(n+1)=\delta^{+}(n)+1$ if a drop exits via the $\operatorname{arm}(1)$. 
. $\delta^{-}(n+1)=\delta^{+}(n)-1$ if a drop exits via the $\operatorname{arm}(2)$.

After a transient regime, $\delta^{-}(n+1)=\delta^{-}(n)$ for all $n$ only if all drops exit the loop by taking either the short arm while $\delta^{-}(n)>0$ or the long arm while $\delta^{-}(n)<0$. The first case implies that the hydrodynamic resistance of the short arm filled with drops is always smaller than that of the long arm: this is the Filter regime. The latter situation cannot be encountered since it requires the number of drops in the long arm to be larger than that of the short arm. Hence, in the Repartition regime, $\delta^{-}(n+1)<\delta^{-}(n)$ when $\delta^{-}(n)>0$ and $\delta^{-}(n+1)>\delta^{-}(n)$ when $\delta^{-}(n)<0$. For any initial conditions, after a transient regime in which $\delta^{-}$evolves monotonically, its sign changes when $\delta^{-}$ reaches either $\epsilon$ or $\epsilon-1$ with $\epsilon=\frac{L_{2}-L_{1}}{L_{d}}-$ floor $\left(\frac{L_{2}-L_{1}}{L_{d}}\right) ; N_{i}$ being integers, $\epsilon$ or $\epsilon-1$ which have opposite signs are the two closest values to 0 that $\delta^{-}$can possibly reach. After the transient regime, one can verify that $\delta^{+}$necessarily fluctuates between these two values. A strict balance between the hydrodynamic resistances of the two arms is unfeasible because of the discrete nature of the drops. As a result, $N_{1}-N_{2}$ is a function of time that can only take two values, $F=$ floor $\left(\frac{L_{2}-L_{1}}{L_{d}}\right)$ or $C=\operatorname{ceil}\left(\frac{L_{2}-L_{1}}{L_{d}}\right)$. In our study, we do not consider the case for which $\frac{L_{2}-L_{1}}{L_{d}}$ is an integer as it is irrelevant experimentally. Since $\frac{R_{2}(t)}{R_{1}(t)}=\frac{Q_{1}(t)}{Q_{2}(t)}=1+\delta(t) /\left(N_{1}+\frac{L_{1}}{L_{d}}\right)$ and $|\delta(t)|<1$, within the limit $N_{1} \rightarrow \infty$ or $L_{1} \rightarrow \infty$, our analysis implies that:

$$
\frac{Q_{1}(t)}{Q_{2}(t)} \rightarrow 1
$$

Hence, within one of the above limit, an equipartition of the flow rates $Q_{1}$ and $Q_{2}$ occurs in steady state as observed numerically and experimentally $[12,13]$.

\section{Repartition regime: Stationary solutions}

Here, we aim to determine the existence of stationary solutions for which the numbers of droplets in both arms remain constant over time. Mathematically, such solutions correspond to $N_{i}^{+}(n+1)=N_{i}^{+}(n)$ for any integer $n$. Using the expressions of $N_{i}^{+}(n)$ given in section II C 1 , it is straightforward to show that $H\left[\delta^{-}(n+\right.$ $1)]=H\left[\delta^{-}\left(n-T_{i}+1\right)\right]$. Hence, when $N_{i}$ is constant over time, $\delta$ is a $T_{i}$-periodic function. Conversely, a $T_{i}$ periodic system implies a constant $N_{i}$. Both $N_{1}$ and $N_{2}$ being constant over time, the system is simultaneously $T_{1}$ - and $T_{2}$-periodic. $T_{1}$ and $T_{2}$ are therefore multiple of the system's period. Furthermore, since in this case $H\left[\delta^{-}(n+1)\right]=H\left[\delta^{-}\left(n-T_{1}+1\right)\right]=H\left[\delta^{-}\left(n-T_{2}+1\right)\right]$, the system is also $\left(T_{2}-T_{1}\right)$-periodic. Such a situation is possible only when $T_{1}$ and $T_{2}$ are multiple of $\left(T_{2}-T_{1}\right)$.

Stationary solutions exist but are only witnessed for specific conditions over the parameters of the problem. Since $N_{1}-N_{2}$ is constant over time, it is therefore equal to either $F$ or $C$. Using Eq. (3) one can then write:

$$
\sum_{j=1}^{T_{1}} N_{2}(j)=\sum_{j=1+T_{1}-T_{2}}^{T_{1}} N_{1}^{H}(j), \text { so that } T_{1} N_{2}=T_{2} N_{1}^{H} .
$$

Using previously established relations, one finds:

$T_{1} N_{2}=T_{2}\left[T_{1}-F-N_{2}\right]$ or $T_{1} N_{2}=T_{2}\left[T_{1}-C-N_{2}\right]$.

Consequently,

$$
N_{2}=\frac{T_{2}}{T_{1}+T_{2}}\left[T_{1}-F\right] \text { or } \quad N_{2}=\frac{T_{2}}{T_{1}+T_{2}}\left[T_{1}-C\right] .
$$

To summarize, a stationary regime occurs whenever either $\frac{T_{2}}{T_{1}+T_{2}}\left[T_{1}-F\right]$ or $\frac{T_{2}}{T_{1}+T_{2}}\left[T_{1}-C\right]$ is an integer. When such a condition is fulfilled, all the quantities remain constant over time and can easily be expressed using relationships derived above.

\section{Repartition regime: Non-stationary solutions}

$N_{1}$ and $N_{2}$ usually evolve over time. However, after a transient regime, numerical simulations show that at least one of these quantities is constant. Since $N_{1}-N_{2}$ only explores two consecutive values, so does the sum $N_{1}+N_{2}$. We assume that $N_{1}+N_{2}$ and $N_{1}-N_{2}$ respectively equal $S$ or $S+1$ and $F$ or $F+1$, where $S$ is an integer and $F=$ floor $\left(\frac{L_{2}-L_{1}}{L_{d}}\right)$. Writing the integers $N_{1}$ and $N_{2}$ as $N_{1}=\frac{N_{1}+N_{2}}{2}+\frac{N_{1}-N_{2}}{2}$ and $N_{2}=\frac{N_{1}+N_{2}}{2}-\frac{N_{1}-N_{2}}{2}$, one shows that:

- When $S$ and $F$ have the same parities, $N_{1}$ fluctuates between $\frac{S+F}{2}$ and $\frac{S+F}{2}+1$ while $N_{2}=\frac{S-F}{2}$ remains constant over time.

- When $F$ and $S$ have different parities, $N_{1}=\frac{S+F+1}{2}$ remains constant over time and $N_{2}$ fluctuates between $\frac{S-F+1}{2}$ and $\frac{S-F-1}{2}$.

To summarize, for non-stationary regimes, the number of drops in one of the two arms is independent of time, the other number fluctuating over time between two consecutive values. $N_{i}$ being constant over time, the dynamics of the system is $T_{i}$-periodic. We have shown that after an initial transient state, periodic dynamics are always obtained, the period being either $T_{1}, T_{2}$ or $T_{2}-T_{1}$.

\section{Repartition regime: Selection of the cycle time}

Here, we derive the selection rules that determine the values taken by one of the invariants of the system, the cycle time $T_{c y c}$ of the binary series.

We begin by considering a $T_{2}$-periodic regime for which $N_{2}=\frac{S-F}{2}$ is constant. Using $\sum_{j=1}^{T_{2}} N_{1}^{H}(j)=T_{1} N_{2}$ established in section II C 3, one finds:

$$
t\left(T_{1}-\frac{S+F}{2}\right)+\left(T_{2}-t\right)\left(T_{1}-\frac{S+F}{2}-1\right)=T_{1} N_{2}
$$


where $N_{1}=\frac{S+F}{2}$ during a cumulative time $t$ and $N_{1}=\frac{S+F}{2}+1$ during $T_{2}-t$. Hence, $t=\left(T_{1}+T_{2}\right) \frac{S-F}{2}-$ $T_{2}\left(T_{1}-F-1\right)$ with $0 \leq t \leq T_{2}$; stationary solutions are obtained when $t=0$ or $t=T_{2}$, both $N_{2}$ and $N_{1}$ remaining constant over time in those cases. The inequality yields:

$$
0 \leq \frac{T_{2}}{T_{1}+T_{2}}\left(T_{1}-F\right)-N_{2} \leq \frac{T_{2}}{T_{1}+T_{2}}<1 .
$$

$N_{2}$ being an integer, the occurrence of a $T_{2}$-periodic solution therefore requires the fractional part of $\frac{T_{2}}{T_{1}+T_{2}}\left(T_{1}-\right.$ $F)$ to be smaller than $\frac{T_{2}}{T_{1}+T_{2}}$. Then $N_{2}$ reads:

$$
N_{2}=\text { floor }\left(\frac{T_{2}}{T_{1}+T_{2}}\left(T_{1}-F\right)\right) \text {. }
$$

Following a similar approach for $T_{1}$-periodic regimes in which $N_{1}=\frac{S+F+1}{2}$ remains constant over time, one finds:

$$
0 \leq \frac{T_{1}}{T_{1}+T_{2}}\left(T_{1}-F\right)-\left(T_{1}-N_{1}\right) \leq \frac{T_{1}}{T_{1}+T_{2}}<1 .
$$

A $T_{1}$-periodic solution can therefore be obtained when the fractional part of $\frac{T_{1}}{T_{1}+T_{2}}\left(T_{1}-F\right)$ is smaller than $\frac{T_{1}}{T_{1}+T_{2}}$. The number of holes in the arm (1), $N_{1}^{H}=T_{1}-N_{1}$, which is constant over time is then given by:

$$
N_{1}^{H}=\text { floor }\left(\frac{T_{1}}{T_{1}+T_{2}}\left(T_{1}-F\right)\right) .
$$

As shown below, the two conditions required for the respective occurrence of $T_{1}$-periodic and $T_{2}$-periodic regimes are incompatible. By noting $p=\frac{T_{1}}{T_{1}+T_{2}}$ and $q=\frac{T_{2}}{T_{1}+T_{2}}$, any integer $M$ can be written as $M=p M+q M$ since $p+q=1$. $I_{p}$ and $\epsilon_{p}$ denoting the integer and fractional parts of $p M$, the integer and fractional parts of $q M$ being $I_{q}$ and $\epsilon_{q}$, one obtains $M=I_{p}+\epsilon_{p}+I_{q}+\epsilon_{q}$.

Two different cases can then be distinguished. When $\epsilon_{p}$ and $\epsilon_{q}$ are both different from zero, $\epsilon_{p}+\epsilon_{q}=1=p+q$. It is then straightforward to derive that $q<\epsilon_{q}<1$ when $0<\epsilon_{p}<p$, and $p<\epsilon_{p}<1$ when $0<\epsilon_{q}<q$. Note that when $\epsilon_{p}=p$ and $\epsilon_{q}=q$, both $p(M-1)$ and $q(M-1)$ are integers. The latter situation corresponds to the condition on $q\left(T_{1}-C\right)$ given Eq. (4) required for a $\left(T_{2}-T_{1}\right)$-periodic regime. When $\epsilon_{p}$ (or $\epsilon_{q}$ ) is equal to zero, then $\epsilon_{q}$ (or $\epsilon_{p}$ ) is also equal to zero. This particular case corresponds to the other condition on $q\left(T_{1}-F\right)$ for the occurrence of a $\left(T_{2}-T_{1}\right)$-periodic regime [see Eq. (4)].

\section{Repartition regime: Selection of the number of packs}

As previously discussed, for a given set of parameters $\left(L_{d}, L_{1}, L_{2}, \lambda\right)$, numerical simulations show that $N_{\text {pack }}$ is an invariant of the problem being independent of initial conditions. For $T_{1}$-periodic regimes, the number of holes in the arm (1) is constant. Since $T_{1}$ is the residence time of a drop or a hole in the arm (1), $N_{\text {pack }}$ is given by $N_{1}^{H}=$ floor $\left(\frac{T_{1}}{T_{1}+T_{2}}\left(T_{1}-F\right)\right)$. Similarly, for $T_{2^{-}}$ periodic regimes, $N_{\text {pack }}$ is given by the constant value of $N_{2}=$ floor $\left(\frac{T_{2}}{T_{1}+T_{2}}\left(T_{1}-F\right)\right)$. For $\left(T_{2}-T_{1}\right)$-periodic regimes, the period is shorter than $T_{1}$ and $T_{2}$, the residence times in the short and long arms, respectively. Using the space-to-time conversion, the emerging cyclic pattern is observed over a portion of the arm (2) having a length $\left(T_{2}-T_{1}\right) \frac{\lambda}{2}$. The number of drops in this portion is $N_{2}-N_{1}^{H}$, both being constant in time. $N_{\text {pack }}$ is then given by either $\frac{T_{2}-T_{1}}{T_{2}+T_{1}}\left(T_{1}-F\right)$ or by $\frac{T_{2}-T_{1}}{T_{2}+T_{1}}\left(T_{1}-C\right)$.

\section{Selection rules}

We summarize below the rules established in previous sections that govern the dynamics of the system. Four cases are identified depending on $x=\frac{T_{2}}{T_{2}+T_{1}}\left(T_{1}-F\right)$ :

- When $x$ is an integer:

$$
T_{c y c}=T_{2}-T_{1} \text { and } N_{p a c k}=\frac{T_{2}-T_{1}}{T_{2}+T_{1}}\left(T_{1}-F\right) ;
$$

- When the fractional part of $x$ is strictly bounded by 0 and $\frac{T_{2}}{T_{2}+T_{1}}$ :

$$
T_{c y c}=T_{2} \text { and } N_{p a c k}=\text { floor }\left(\frac{T_{2}}{T_{1}+T_{2}}\left(T_{1}-F\right)\right) ;
$$

- When the fractional part of $x$ is equal to $\frac{T_{2}}{T_{2}+T_{1}}$ :

$$
T_{c y c}=T_{2}-T_{1} \text { and } N_{p a c k}=\frac{T_{2}-T_{1}}{T_{2}+T_{1}}\left(T_{1}-C\right) ;
$$

- When the fractional part of $x$ is strictly bounded by $\frac{T_{2}}{T_{2}+T_{1}}$ and 1 :

$$
T_{c y c}=T_{1} \text { and } N_{p a c k}=\text { floor }\left(\frac{T_{1}}{T_{1}+T_{2}}\left(T_{1}-F\right)\right) \text {. }
$$

The invariants are indeed independent of the number and positions of the drops initially present in the loop and solely depend on $L_{d}, L_{1}, L_{2}$, and $\lambda$. As shown in Fig. 3 and Fig. 4, these theoretical predictions for the evolutions of $T_{c y c}$ and $N_{\text {pack }}$ concur well with numerical simulations. They predict the occurrence of the various plateaus obtained numerically and the numerous bifurcations between these plateaus as $\lambda$ varies [13]. Between two successive plateaus, we note the emergence of singular periodic regimes with very long cycle times. These regimes which are not predicted by our model only exist for very narrow ranges of $\lambda$, narrower than the experimental stochastic noise of $\lambda$, are not observable experimentally [13].

Using these predictions, one may also derive analytical expressions for the third invariant of the system, $F_{2}=\frac{N_{\text {pack }}}{T_{c y c}}$, the fraction of droplet flowing through the 

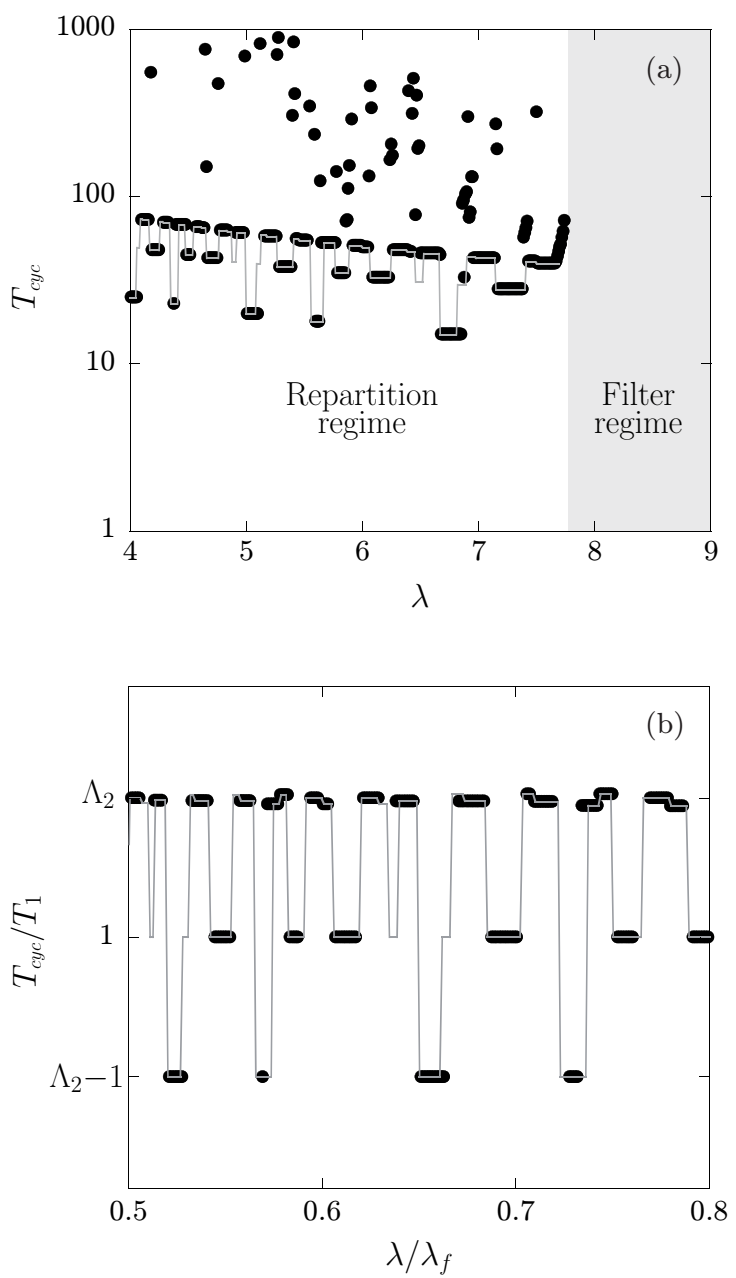

FIG. 3. (a) $T_{c y c}$ versus $\lambda$ for $L_{1}=100, L_{2}=150$, and $L_{d}=2$. The lengths have arbitrary units. The solid line is calculated using the discrete model. Closed circles stand for numerical results. (b) Bifurcation diagrams of $T_{c y c} / T_{1}$ as a function of $\lambda / \lambda_{f}$ shown in the range $\lambda / \lambda_{f}=0.5-0.8$.

long arm. When comparing predictions of the continuous (Fig. 2) and discrete (see Fig. 5) models, the numerical results are better described by the latter model.

A simple criterion permits to predict the values of $\lambda$ for which a bifurcation between different periodical regimes may occur [13]. As $\lambda$ varies, one expects a change of dynamical behavior whenever the integers $T_{1}$ or $T_{2}$ change by 1 . This occurs whenever $\lambda=\lambda_{c}(i, k)$ with:

$$
\frac{2 L_{i}}{\lambda_{c}(i, k)}=\text { floor }\left(\frac{L_{2}-L_{1}}{L_{d}}\right)+k,
$$

where $i=1,2$ and $k \in \mathbb{N}^{\star}$. Although this simple criterion overestimates the number of observed bifurcations [13], it predicts the exact value of $\lambda=\lambda_{f}$ at which the transition between Filter and Repartition regimes occurs:

$$
\lambda_{f}=\frac{2 L_{1}}{\text { floor }\left(\frac{L_{2}-L_{1}}{L_{d}}\right)+1} .
$$

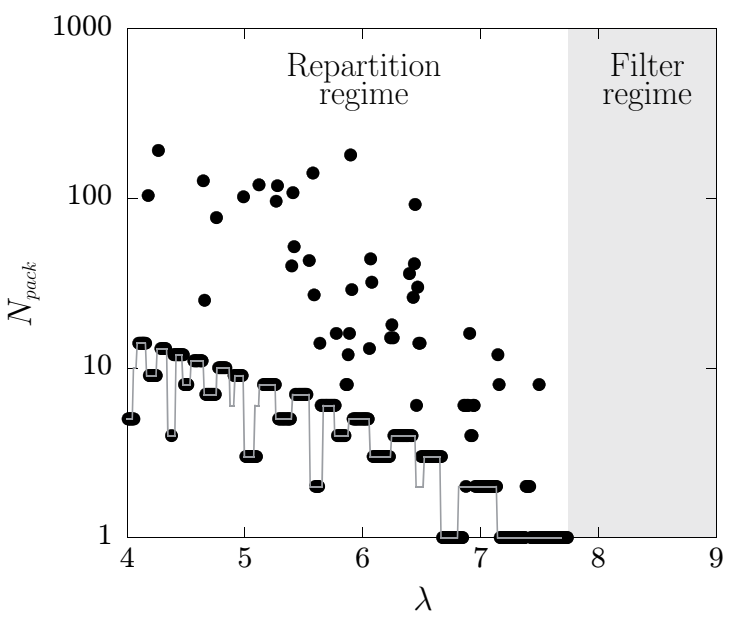

FIG. 4. $N_{\text {pack }}$ versus $\lambda$ for $L_{1}=100, L_{2}=150$, and $L_{d}=2$. The lengths have arbitrary units. The solid line is calculated using the discrete model. Closed circles stand for numerical results.

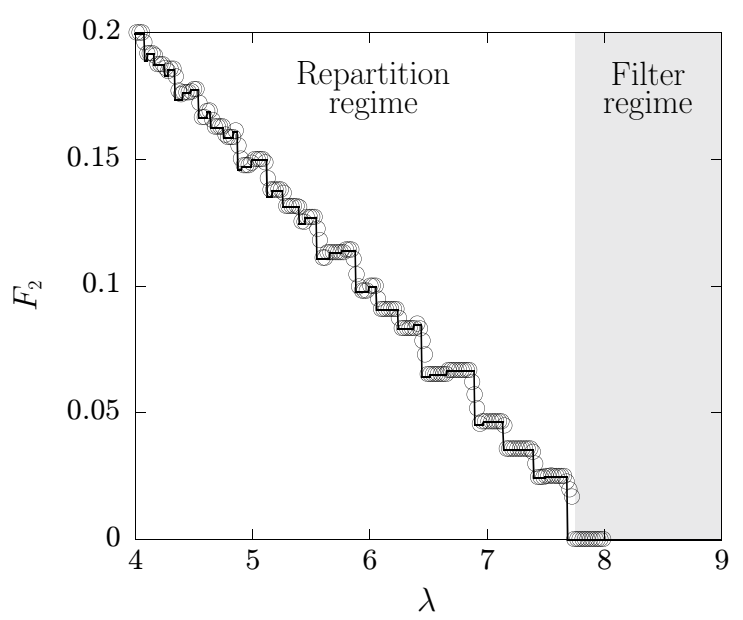

FIG. 5. $\quad F_{2}$ versus $\lambda$ for $L_{1}=100, L_{2}=150$, and $L_{d}=2$. The lengths have arbitrary units. Numerical results (circles) are compared with the prediction of the discrete model (solid line), calculated using $F_{2}=N_{\text {pack }} / T_{c y c}$.

\section{Connections between the two models}

When $L_{1}, L_{2}$ and $L_{2}-L_{1}$ are large compared to $L_{d}$, one can write $F \sim C \sim \frac{L_{1}\left(\Lambda_{2}-1\right)}{L_{d}}$ and $T_{i} \sim \frac{2 L_{i}}{\lambda}$. Within this limit, using Eq.(6) and the selections rules previously established, it is straightforward to show that $\lambda_{f} \sim \frac{2 L_{d}}{\Lambda_{2}-1}$ and $F_{2} \sim \frac{1}{\Lambda_{2}+1}\left[1-\lambda \frac{\Lambda_{2}-1}{2 L_{d}}\right]$. These mathematical expressions for the predictions of $\lambda_{f}$ and $F_{2}$ are identical to those found using the continuous model [12]. 


\section{COMPLEX NETWORKS: TWO EMBEDDED LOOPS}

\section{A. Numerical algorithm and steady state results}

We now study the flow of a periodic train of droplets in two embedded loops made of four arms of different lengths and two inlet nodes $A$ and $B$ (Fig. 6); channels have identical widths and $L_{1}<L_{2}$.

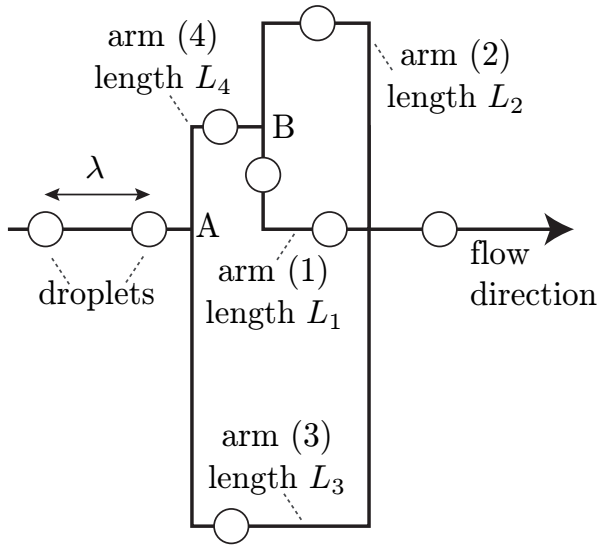

FIG. 6. Schematic of the flow model for two imbedded loops having two inlet nodes $A$ and $B$. Defined are $\lambda$ and $L_{i}$ with $i=1,2,3$, and 4 , and $L_{1}<L_{2}$.

Our simulations are based on an algorithm similar to the one used in the study of a single asymmetric loop described in section II. Drops periodically feed the inlet node $A$ at a rate $f$. Whenever a drop reaches either $A$ or $B$, the number of drops $N_{i}$ and the hydrodynamic resistance $R_{i}$ are computed. The drop located at one of the inlet node is then injected in the branch having the largest flow rate. Afterwards, the values of $N_{i}, R_{i}$, and $V_{i}$ are updated and all drops are moved until $N_{i}$ changes. The whole network does not contain any drops when simulations begin. After a transient state, several hydrodynamic regimes are observed when $\lambda$ varies. As shown below, an important parameter is the hydrodynamic resistance of the branch formed by the arms (1), (2), and (4). This resistance is identical to that of a single arm having a length $L_{e q}=L_{4}+L_{1} L_{2} /\left(L_{1}+L_{2}\right)$. For a given $\lambda$, the observed regime depends on whether $L_{e q}>L_{3}$.

When $L_{e q}>L_{3}$, we observe the following sequence of regimes when $\lambda$ decreases (see Fig. 7):

- $\lambda>\lambda_{f}^{(1)}$ : all droplets flow through the arm (3);

- $\lambda_{f}^{(1)}>\lambda>\lambda_{f}^{(2)}$ : drops explore both arms (3) and (1);

- $\lambda_{f}^{(2)}>\lambda$ : the droplets flow through all the arms.

When $L_{e q}<L_{3}$, we observe the following sequence (Fig. 8):

- $\lambda>\lambda_{f}^{(1)}$ : all droplets flow through the arm (1);

- $\lambda_{f}^{(1)}>\lambda>\lambda_{f}^{(2)}$ : drops only flow in arms (1) and (2);

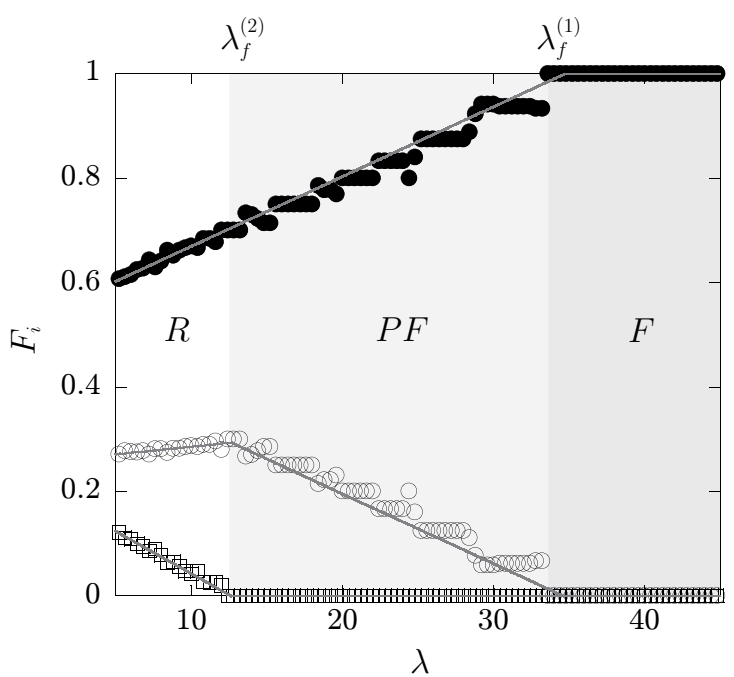

FIG. 7. Variations of $F_{1}(\circ), F_{2}(\square)$, and $F_{3}(\bullet)$ with $\lambda$. The parameters expressed in arbitrary units are $L_{1}=L_{3}=100$, $L_{2}=125, L_{4}=60$, and $L_{d}=2.7\left(L_{e q}>L_{3}\right)$. The solid lines are predictions calculated using the continuous model (see section III B). $R, P F$, and $F$ respectively denote the Repartition, Partial Filter, and the Filter regimes.

- $\lambda_{f}^{(2)}>\lambda$ : the droplets explore all arms.

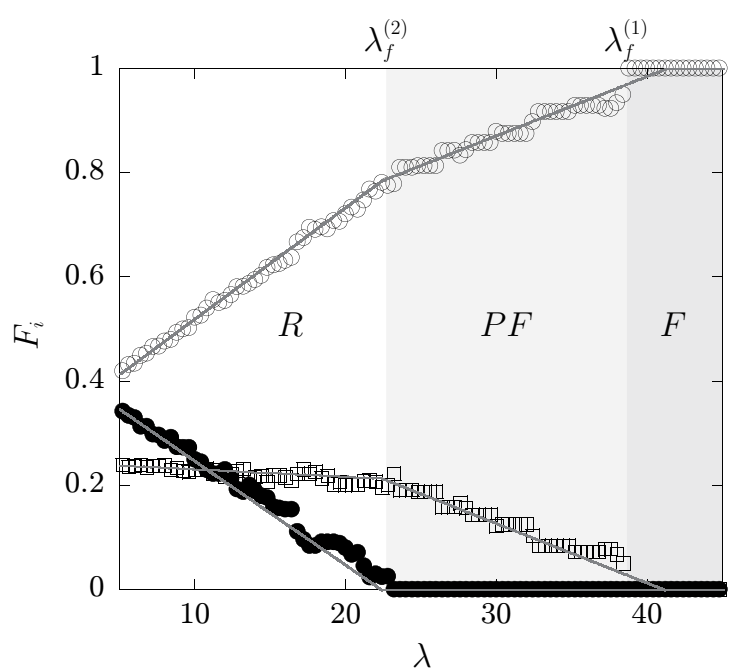

FIG. 8. Variations of $F_{1}(\circ), F_{2}(\square)$, and $F_{3}(\bullet)$ with $\lambda$. The parameters expressed in arbitrary units are $L_{1}=L_{3}=100$, $L_{2}=125, L_{4}=25$, and $L_{d}=2.7\left(L_{e q}<L_{3}\right)$. The solid lines are calculated using the continuous model (see section IIIB).

The critical dilutions $\lambda_{f}^{(1)}$ and $\lambda_{f}^{(2)}$ separating the three observed hydrodynamic regimes depend on the parameters of the problem, $\left[L_{i}, L_{d}\right]$. As shown below, mathematical expressions for these quantities depend on whether or not $L_{e q}>L_{3}$, both cases presenting similar features.

As $\lambda$ decreases the number of selected paths increases until $\lambda<\lambda_{f}^{(2)}$, a region where all possible paths are ex- 
plored: this is the Repartition regime (denoted $R$ ). When $\lambda_{f}^{(1)}<\lambda$, only one path is taken: this is the Filter regime (denoted $F$ ) in which all drops flow in the arm having the smallest hydrodynamic resistance in the absence of drops. For intermediate dilutions, that is, for $\lambda_{f}^{(2)}<\lambda<\lambda_{f}^{(1)}$, the droplets only explore two out of the three possible paths, we next refer to this regime as the Partial Filter regime (denoted $P F$ ). As $\lambda$ decreases, the paths in which drops flow are selected according to the ascending order of their hydrodynamic resistances in the absence of drops.

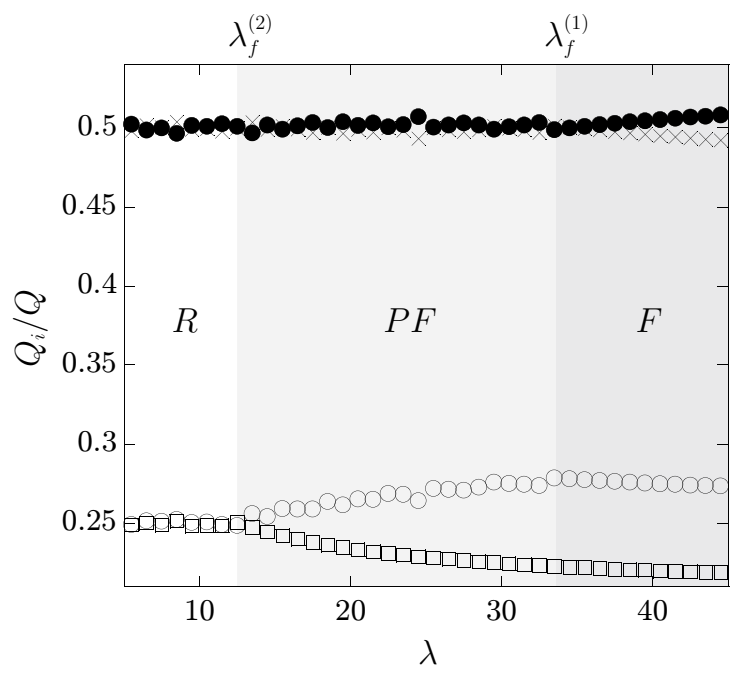

FIG. 9. Variations of $Q_{i} / Q$ with $\lambda, Q_{i}$ and $Q$ being the total flow rates in the arm $(i)$ and in the whole circuit. The symbols $(\times),(\bullet),(\square)$, and (०) respectively correspond to $i=4$, 3,2 , and 1 . The parameters expressed in arbitrary units are: $L_{1}=L_{3}=100, L_{2}=125, L_{4}=60$, and $L_{d}=2.7\left(L_{e q}>L_{3}\right)$.

At any node of the network where the drops divide between two arms, the total flow rates in each of these arms are nearly equal (Fig. 9 and Fig. 10). Such features seem inherent to any dynamics of droplet traffic in singlelane microfluidic networks since they can be observed in other complex circuits (results not shown here).

\section{B. Interpretation using the continuous approach}

Our numerical findings can be understood using the continuous approach employed for single asymmetric loops (section II B). Similarly to that case, we define the mean frequency $f_{i}$ characterizing the entrance (or exit) of drops in the arm $(i)$ and the mean distance $\lambda_{i}$ between two consecutive drops in this arm; we use the variable $X_{i}=1 / \lambda_{i}$ for readability.

In the Repartition regime, using the equipartition of the total flow rate and the conservation of the dispersed phase at nodes $A$ and $B$, one derives two equations: $X_{3}+X_{4}=2 / \lambda$ and $X_{1}+X_{2}=2 X_{4}$. Writing the equality of the pressure drops between the ends of arms (1) and (2) and between the extremities of the arm (3) and the ends

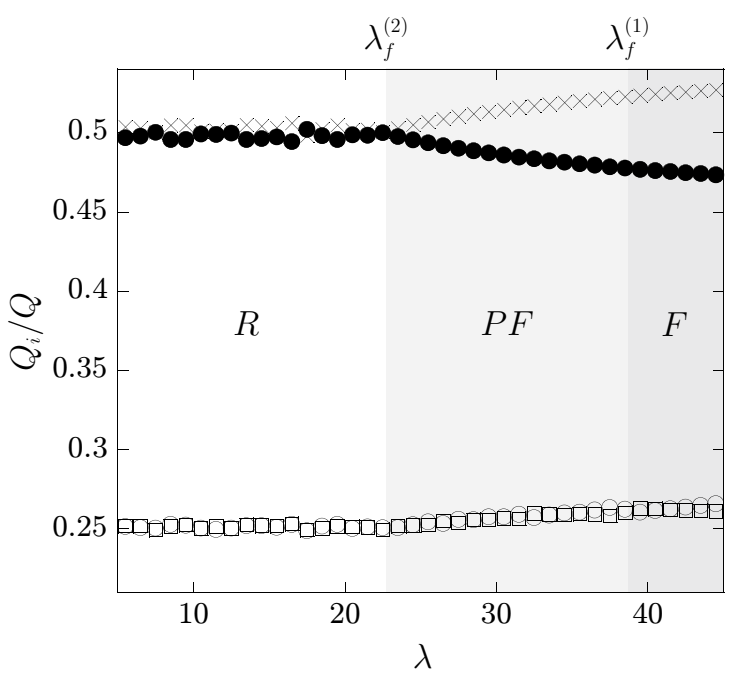

FIG. 10. Variations of $Q_{i} / Q$ with $\lambda$, where $Q_{i}$ and $Q$ are the total flow rates in the arm $(i)$ and in the whole circuit. The symbols $(\times),(\bullet),(\square)$, and (o) respectively correspond to $i=4$, 3,2 , and 1 . The parameters expressed in arbitrary units are: $L_{1}=L_{3}=100, L_{2}=125, L_{4}=25$, and $L_{d}=2.7\left(L_{e q}<L_{3}\right)$.

of the branch formed by arms (4) and (1), and using the equipartition of the total flow rate at the two nodes, two other equations can be obtained: $\Lambda_{2}\left(1+L_{d} X_{2}\right)=1+L_{d} X_{1}$ and $\Lambda_{3}\left(1+L_{d} X_{3}\right)=\Lambda_{4}\left(1+L_{d} X_{4}\right)+\left(1+L_{d} X_{1}\right) / 2$, where $\Lambda_{i}=\frac{L_{i}}{L_{1}}$. By solving this set of four linear equations, one finds the mathematical expression for $X_{i}$. Then, analytical expressions for both $f_{i}$ and the fractions $F_{i}=f_{i} / f$ of drops flowing in the arm $(i)$ are obtained by using the equipartition of the total flow rate at the nodes $A$ and $B$. One easily finds $f_{4}=X_{4} \lambda f / 2, f_{3}=X_{3} \lambda f / 2, f_{1}=X_{1} \lambda f / 4$, and $f_{2}=X_{2} \lambda f / 4 ; f_{i}$ is independent of $L_{e q} / L_{3}$.

The analytical expression for $F_{i}$ can also be found in the Partial Filter regime using the continuous approach. However, these expressions which we next derive depend on whether $L_{e q}>L_{3}$ or $L_{e q}<L_{3}$, as the two selected paths are different in the two cases.

When $L_{e q}<L_{3}$, for $\lambda_{f}^{(1)}>\lambda>\lambda_{f}^{(2)}$, all drops flow either in the $\operatorname{arm}(1)$ or in the $\operatorname{arm}(2)$, i.e. $F_{3}=0$ and all drops reaching the node $A$ flow in the arm (4) (see Fig. 6). The droplets are therefore fed in the asymmetric loop made of the arms (1) and (2) at the rate $f$. Then the train of drops reaching the node $B$ is also periodic in space, but its period $\widetilde{\lambda}$ is no longer $\lambda$ since some continuous phase flows in the arm (3) at the node $A$. Hence, the expressions derived for $F_{2}$ and $F_{1}=1-F_{2}$ when drops divide in single asymmetric loops are still valid provided that one replaces $\lambda$ by $\tilde{\lambda}$ in Eq. (1a). In what follows, we determine the needed relation between $\widetilde{\lambda}$ and $\lambda$. The total flow rates at the nodes $A$ and $B$ are $Q=S \lambda f / \beta$ and $q=S \widetilde{\lambda} f / \beta$, respectively. Using the equipartition rule at the node $B$, the total flow rate in the arm (1) is $q / 2$. Writing the equality of the pressure drops of the arm (3) and the serial association of arms (1) and (4) and using 
the conservation of the total flow rates at the node $A$, one finds:

$$
\widetilde{\lambda}\left(\Lambda_{4}+\Lambda_{3}+\frac{\Lambda_{2}}{1+\Lambda_{2}}\right)=\Lambda_{3} \lambda-L_{d}\left(\Lambda_{4}+\frac{\Lambda_{2}}{1+\Lambda_{2}}\right)
$$

When $L_{e q}>L_{3}, F_{2}=0$ in the Partial Filter regime, so that all drops flow either in the arm (1) or in the arm (3) and $f_{1}=f_{4}$ (see Fig. 6). Using the equipartition rule at the node $A$ and the conservation of the total flow rate at the node $B$, one finds the total flow rates in arms (3) and (4), $Q / 2=S \lambda f / 2 \beta=S \lambda_{4} f_{4} / \beta=S \lambda_{3} f_{3} / \beta$. Also, in arms (1) and (2), the flow rates are $q=S \lambda_{1} f_{1} / \beta$ and $Q / 2-$ $q$, respectively. Using the equality of the pressure drops of the arm (1) filled with droplets and the arm (2) in the absence of drops, one shows $\lambda_{1}=\left(\lambda_{4} \Lambda_{2}-L_{d}\right) /\left(1+\Lambda_{2}\right)$. By using the equality of the pressure drops of the arm (3) and the serial association of arms (1) and (4), the conservation of the total flow rate, and the conservation of the dispersed phase at the node $A, F_{1}$ reads:

$$
F_{1}=\frac{2 L_{d} \Lambda_{3}-\lambda\left[\Lambda_{4}-\Lambda_{3}+\Lambda_{2} /\left(1+\Lambda_{2}\right)\right]}{2 L_{d}\left[\Lambda_{3}+\Lambda_{4}+\Lambda_{2} /\left(1+\Lambda_{2}\right)\right]} .
$$

One then easily finds $F_{3}=1-F_{1}$ and $F_{4}=F_{1}$. As shown in Fig. 7 and Fig. 8, the predictions concur well with numerical results both for $L_{e q}<L_{3}$ and $L_{e q}>L_{3}$.

\section{Dynamics: a restricted discrete approach}

We now study the dynamical properties of the system. The drops are indexed when entering the network (node $A$ ), and their successive path selections are coded into series of $-1,0$, and 1 when they flow in the arm (3), (2), and (1), respectively. Our numerical simulations reveal that in the Repartition regime, in contrast to the Partial Filter regime, the cycle time of the corresponding signals depends on initial conditions, i.e. the number and positions of droplets initially present in the circuit. For the sake of simplicity, in both cases $L_{e q}<L_{3}$ and $L_{e q}>L_{3}$, we will discuss results for which no droplets are present in the network when simulations start.

We first study the case $L_{e q}<L_{3}$. Figure 11 shows the variations of the period of the signals describing the successive selected paths with $\lambda$. In the Partial Filter regime, one observes a succession of plateaus where the period is constant, separated by bifurcations. In the narrow regions between two successive plateaus, the period is unusually large, much larger than the different residence times of the drops in the network; such regimes irrelevant to experiments are also observed in the case of single loops (section II C 7). In the Partial Filter regime, all drops flow in either the arm (1) or the arm (2). Similarly to our modeling in the previous section, replacing $\lambda$ by $\widetilde{\lambda}$ and using Eq. (7), we can use the selection rules derived in section II C 7. One obtains a relatively good description of the dynamics in this regime (see Fig. 12). The value of $T_{c y c}$ for a plateau and the transition between plateaus are well predicted. Since $F_{2}=N_{\text {pack }} / T_{c y c}$

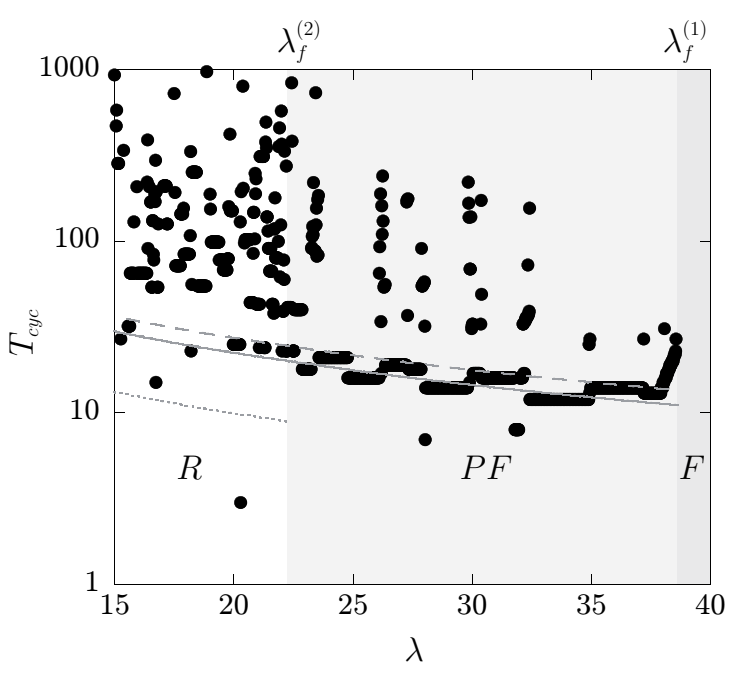

FIG. 11. Variations of $T_{c y c}$ with $\lambda$. The parameters expressed in arbitrary units are: $L_{1}=L_{3}=100, L_{2}=125, L_{4}=25$ and $L_{d}=2.7\left(L_{e q}<L_{3}\right)$. The dotted, dashed, and solid lines correspond to the mean travel times of drops exiting by the arm (3), (2), and (1), respectively. These times are computed using the continuous approach.

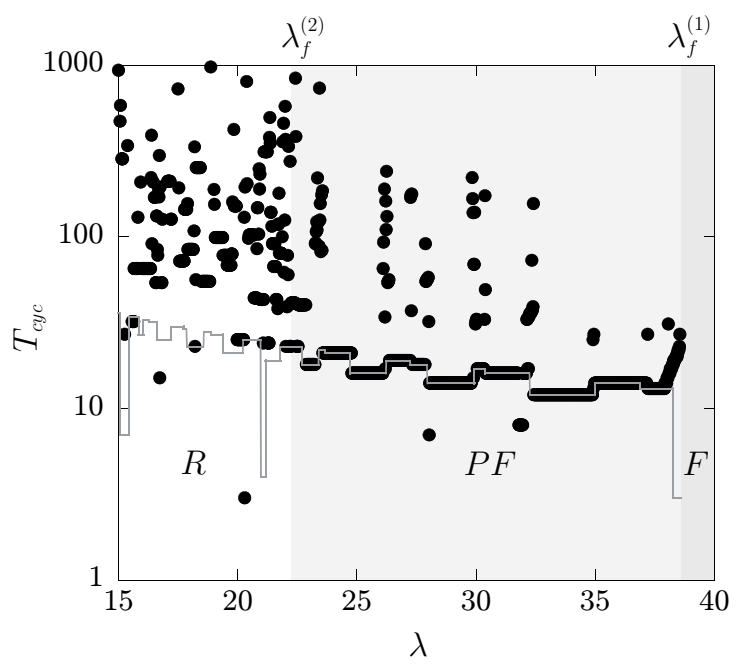

FIG. 12. Shown is $T_{c y c}$ versus $\lambda$ for $L_{1}=L_{3}=100, L_{2}=125$, $L_{4}=25$, and $L_{d}=2.7$ expressed in arbitrary units $\left(L_{e q}<L_{3}\right)$. The solid lines is calculated using the expressions derived with the discrete approach for which $\lambda$ is replaced by $\widetilde{\lambda}$.

in the Partial Filter regime, this approach also permits to model the variations of $F_{2}$ and $F_{1}=1-F_{2}$ with $\lambda$ in this regime (Fig. 13). The resulting predictions provide a better description of the fraction of drops than the continuous model (see Fig. 8).

We now investigate the case $L_{e q}>L_{3}$. Figure 14 shows the period of the signals as a function of $\lambda$. Both in Repartition and Partial Filter regimes, we also obtain a succession of bifurcations between different plateaus. As observed for single and imbedded loops when $L_{e q}<L_{3}$, 


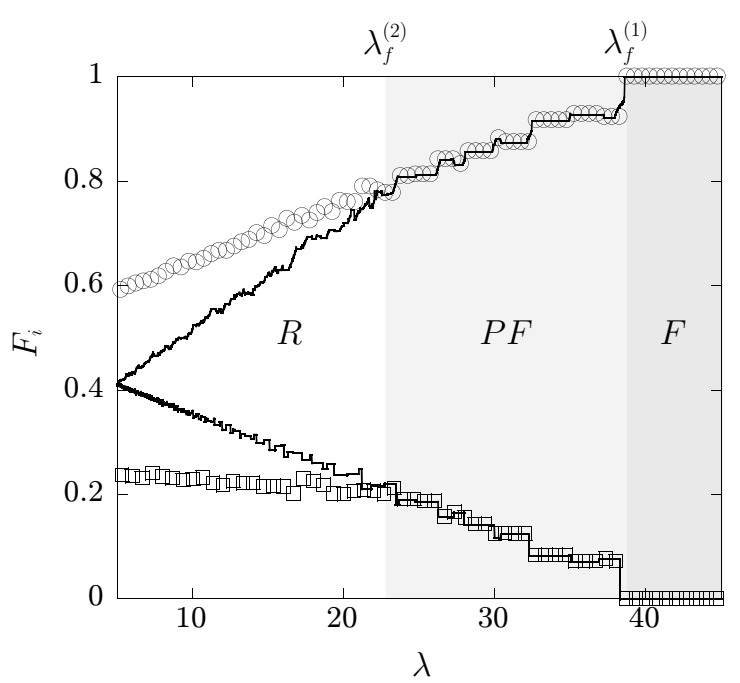

FIG. 13. Variations of $F_{1}$ (०) and $F_{2}(\square)$ with $\lambda$ for $L_{1}=L_{3}=100, L_{2}=125, L_{4}=25$, and $L_{d}=2.7\left(L_{e q}<L_{3}\right)$. The solid lines are calculated using the discrete model in the Partial Filter regime; these predictions that are extended to the other regimes are established by replacing $\lambda$ by $\tilde{\lambda}$.

irrelevant regimes with unusually long cycle times appear in the narrow regions between successive plateaus. In the Partial Filter regime, droplets only flow in either the arm (1) or the arm (3). However, in contrast to the case of a single asymmetric loop, the temporal fluctuations of the number of drops, thus those of the holes, present in the two selected paths can be larger than one. This difference results from the presence of node $B$ which provides an extra degree of freedom to this system. The exit and entrance of drops in the arm (1) alter the total flow rate in the arm (2), which in turns modify the velocity of the droplets traveling in arms (1) and (4). For this reason, in contrast to the case $L_{e q}<L_{3}$, one cannot employ the discrete approach to find simple selections rules.

\section{Experiments}

To validate our numerical predictions, we carry out experiments with planar microfluidic devices made of polydimethylsiloxane (PDMS) and fabricated using standard soft lithography techniques [35] (see Fig. 15).

A periodic train of monodisperse water-in-oil droplets is produced in a flow focusing geometry [29]. The drop size $D$ and the production rate $f$ are controlled by the flow rates $Q_{c}^{f}$ and $Q_{d}$ of the continuous and dispersed phases, respectively (Fig. 15). Additional volumes of the continuous phase can be infused or withdrawn downstream the production module by changing the flow rate $Q_{c}^{d}$ in a dilution module (Fig. 15). Adjustments of $Q_{c}^{d}$ permit to vary the velocity of the droplets $V$, i.e. the dilution $\lambda=V / f$, while $D$ and $f$ remain constant [25]. The drops are then directed towards the inlet of two imbedded

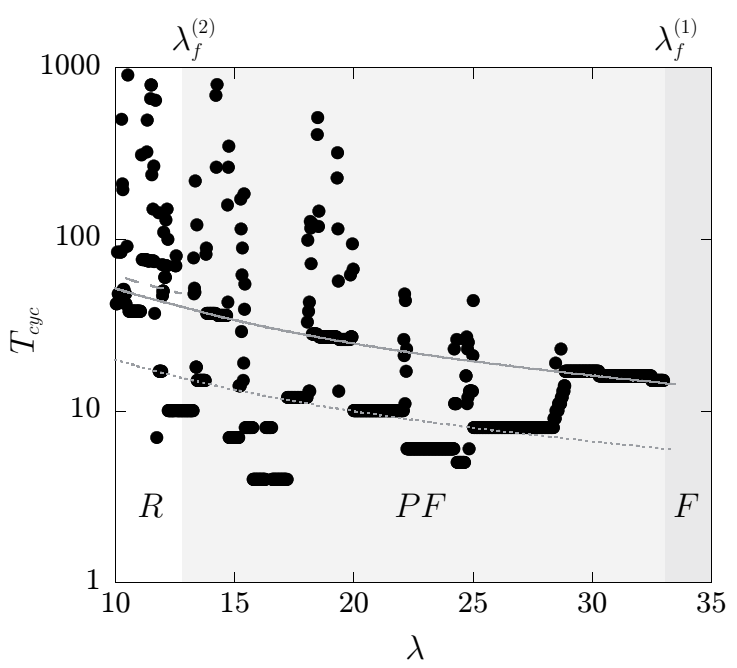

FIG. 14. Shown is $T_{c y c}$ versus $\lambda$ for $L_{1}=L_{3}=100, L_{2}=125$, $L_{4}=60$, and $L_{d}=2.7\left(L_{e q}>L_{3}\right)$. The dotted, dashed, and solid lines correspond to the mean travel times of drops exiting by the arm (3), (2), and (1), respectively. These times are computed using the continuous approach.

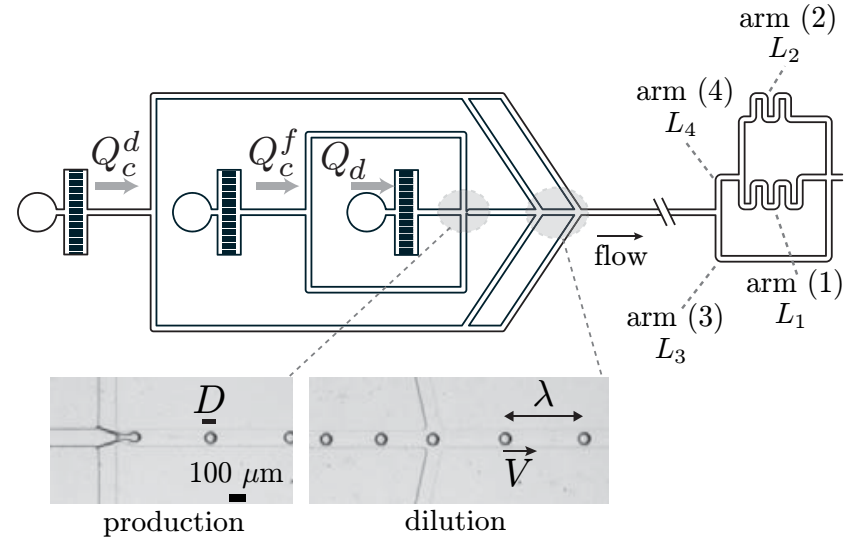

FIG. 15. Experiments: Schematic of the microfluidic device and photographs defining $D, \lambda$, and $V$.

loops (Fig. 15); this network is similar to the one depicted in Fig. 6. The dispersed and continuous phases consist of a mixture of water containing $15 \mathrm{~g} / \mathrm{L}$ of sodium dodecyl sulfate (SDS) and hexadecane, respectively. Videos of the flow are recorded with a fast camera (Phantom V7) typically working at 1000 frames/s. $D, V, \lambda$, as well as the trajectory of each drop in the network are obtained from image analysis using a custom-written software developed with MATLAB. In all experiments, the Reynolds and the capillary numbers are very small and span the ranges $10^{-3}-10^{-1}$ and $10^{-3}-10^{-2}$, respectively. For this range of capillary numbers and any values of $D$, we do not observe droplet breakup nor collision between drops at any $\mathrm{T}$ junctions of the circuit $[21,25,36-40]$.

Figure 16 shows the three hydrodynamic regimes 


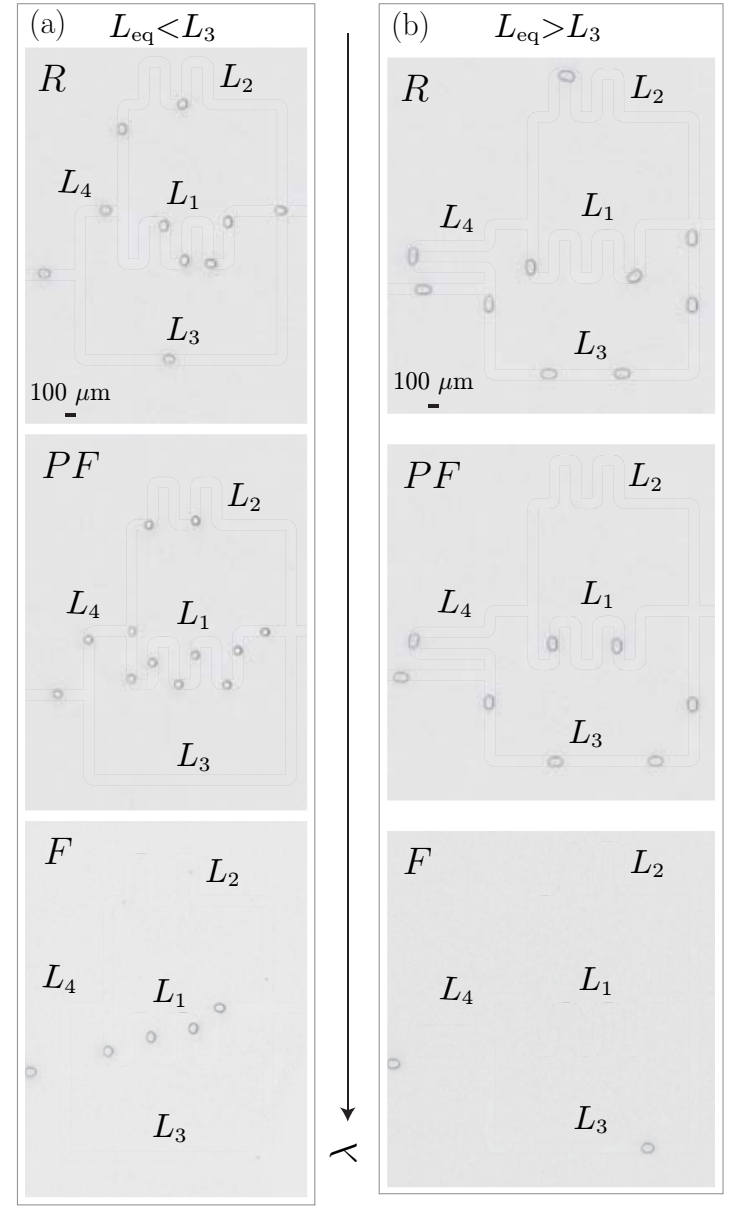

FIG. 16. Images of the three hydrodynamic regimes observed in imbedded loops when varying $\lambda$ for $\left(\mathrm{a}, L_{e q}<L_{3}\right)$ $L_{1}=L_{3}=4 \mathrm{~mm}, L_{2}=5 \mathrm{~mm}, L_{4}=1 \mathrm{~mm}$ and (b, $\left.L_{e q}>L_{3}\right)$ $L_{1}=L_{3}=4 \mathrm{~mm}, L_{2}=5 \mathrm{~mm}, L_{4}=2.6 \mathrm{~mm}$. The inlet and outlet of the loop are respectively located on the left and on the right of each image.

found experimentally as $\lambda$ varies when either $L_{e q}<L_{3}$ or $L_{e q}>L_{3}$. In both cases, at large dilutions, all drops flow through one arm. As the dilution decreases, the drops explore two and eventually three paths. As shown in Fig. 16, the sequence of selected paths depends on whether $L_{e q}<L_{3}$ or $L_{e q}>L_{3}$. In each case, the observed sequence concurs with numerical and theoretical findings. As suggested by our simulations, as $\lambda$ decreases, the paths are selected according to the ascending order of their hydrodynamic resistance in the absence of drops.

We study the variations of the droplet fraction $F_{i}$ in each arm (i) with $\lambda$ for $L_{e q}>L_{3}$ and constant values of $D$ and $f$. Comparing the results to predictions calculated using the continuous approach, $L_{d}$ being the only free parameter, we obtain a relatively good agreement (Fig. 17). This indicates that the continuous model can describe droplet traffic in complex single-lane networks.

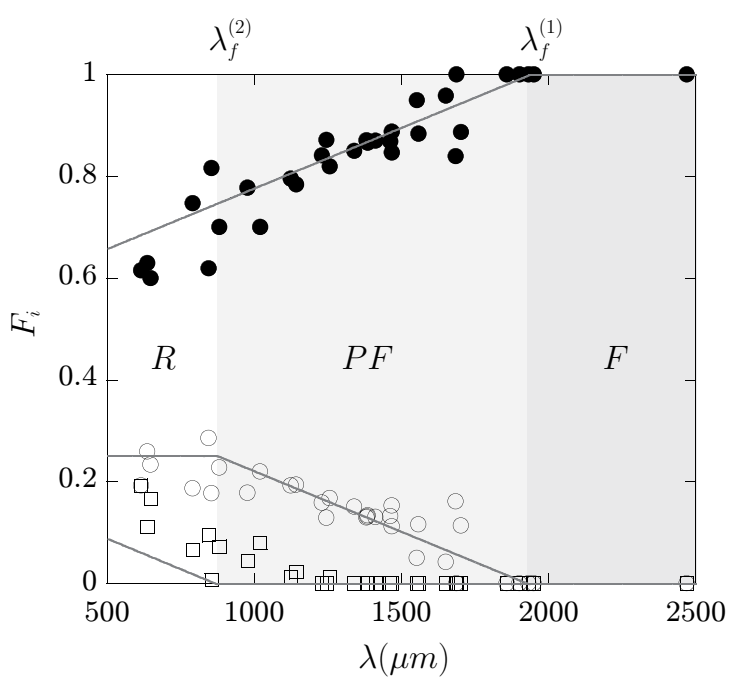

FIG. 17. Experimental variations of $F_{1}(\circ), F_{2}(\square)$, and $F_{3}$ $(\bullet)$ with $\lambda$ in the configuration shown in Fig. 16(b). The solid lines are calculated using the continuous approach with $L_{d}=165 \mu \mathrm{m}$ in the Repartition and Partial Filter regime.

\section{CONCLUSION}

We have investigated the flow of periodic trains of monodisperse droplets through various single-lane networks. At steady state, when drops divide at a node between two arms, our results show that the mean total flow rates in these arms are nearly equal for any topology of the network. Using this generalized Equipartition rule, we have demonstrated that the continuous modeling approach, introduced to describe traffic through single asymmetric loops $[10,12,14,16]$, can be successfully adapted for more complex single-lane networks. This approach which neglects the discrete nature of droplets gives a good description of the steady states provided that the temporal fluctuations of the hydrodynamic resistances of the various arms can be neglected; such fluctuations result from the entrance and exit of drops in these arms. In the case of two imbedded loops, our study reveals the existence of three hydrodynamic regimes as the dilution varies, each regime being characterized by the number of paths explored by the drops. Above a critical dilution, the droplets only take the path that has the smallest hydrodynamic resistance in the absence of drops: this is the Filter regime. As the dilution decreases, the number of explored paths increases and eventually, the whole network contains droplet in the so-called Repartition regime. As the dilution decreases, the paths are selected according to the ascending order of their hydrodynamic resistance in the absence of drops.

The dynamics of droplet traffic in microfluidic networks is controlled by time delayed feedbacks as a path selection depends on the paths taken by the preceding drops. Complex dynamical behaviors result from such feedbacks, notably long-lasting periodic states separated 
by a wealth of bifurcations as the dilution varies. In contrast to the case of a single asymmetric loop, the period of the system may strongly depend on initial conditions. This sensitivity to initial conditions is due to extra degrees of freedom, related to the presence in the network of more than one node where the drops select the path to take. In the Partial Filter regime in which the drops only explore two paths, depending on the topology of the network, we have shown that the discrete approach developed by Sessoms et al. [13] in the context of asymmetric loops can successfully describe the dynamics. Such an approach is possible provided that downstream the node where the drops divide, no bifurcations exist along the two possible paths.

\section{ACKNOWLEDGMENTS}

This work was partially funded by an ACOMB grant (Droplets) obtained from the Brittany region of France, a PEPS grant from CNRS, and the Université de Bretagne (EPT Physfood). We acknowledge fruitful discussions with D. A. Sessoms and we thank J. Bonte and G. Jézéquel for preliminary numerical and experimental studies.
[1] H. Bruus, Theoretical Microfluidics (Oxford University Press, New-York, 2008).

[2] A. Ajdari, C. R. Phys. 5, 539 (2004).

[3] K. W. Oh, K. Lee, B. Ahn, and E. P. Furlani, Lab. Chip 12, 515 (2012).

[4] One can introduce capacitors in the analog electric circuits to account for the deformability of the conducts.

[5] M. Joanicot and A. Ajdari, Science 309, 887 (2005).

[6] N. Lorber, F. Sarrazin, P. Guillot, P. Panizza, A. Colin, B. Pavageau, C. Hany, P. Maestro, S. Marre, T. Delclost, C. Aymonier, P. Subra, L. Prat, C. Gourdon, and E. Mignard, Lab. Chip 11, 779 (2011).

[7] K. Nagel, Phys. Rev. E 53, 4655 (1996).

[8] R. T. Carr, and M. Lacoin, Ann. Biomed. Eng. 28, 641 (2000).

[9] D. Angeli, J. E. Ferrell, and E. D. Sontag, Proc. Natl. Acad. Sci. U.S.A. 101, 1822 (2004).

[10] W. Engl, M. Roche, A. Colin, P. Panizza, and A. Ajdari, Phys. Rev. Lett. 95, 208304 (2005).

[11] F. Jousse, R. Farr, D. R. Link, M. J. Fuerstman, and P. Garstecki, Phys. Rev. E 74, 036311 (2006).

[12] D. A. Sessoms, M. Belloul, W. Engl, M. Roche, L. Courbin, and P. Panizza, Phys. Rev. E 80, 016317 (2009).

[13] D. A. Sessoms, A. Amon, L. Courbin, and P. Panizza, Phys. Rev. Lett. 105, 154501 (2010).

[14] T. Glawdel, C. Elbuken, and C. L. Ren, Lab. Chip 11, 3774 (2011).

[15] M. J. Fuerstman, P. Garstecki, and G. M. Whitesides, Science 315, 828 (2007).

[16] O. Cybulski and P. Garstecki, Lab. Chip 10, 484 (2010).

[17] M. Schindler and A. Ajdari, Phys. Rev. Lett. 100, 044501 (2008).

[18] V. Labrot, M. Schindler, P. Guillot, A. Colin, and M. Joanicot, Biomicrofluidics 3, 012804 (2009).

[19] B. J. Smith and D. P. Gaver III, Lab. Chip, 10, 303 (2010).

[20] M. D. Behzad, H. Seyed-allaei, and M. R. Ejtehadi, Phys. Rev. E, 82, 037303 (2010).

[21] M. Belloul, L. Courbin, and P. Panizza, Soft Matter 7, 9453 (2011).
[22] P. Parthiban and S. A. Khan, Lab. Chip 12, 582(2012).

[23] W. Choi, M. Hashimoto, A. K. Ellerbee, X. Chen, K. J. M. Bishop, P. Garstecki, H. A. Stone, and G. M. Whitesides, Lab. Chip 11, 3970 (2011).

[24] R. Jeanneret, J. P. Vest, and D. Bartolo, Phys. Rev. Lett. 108, 034501 (2012).

[25] M. Belloul, W. Engl, A. Colin, P. Panizza, and A. Ajdari, Phys. Rev. Lett. 102, 194502 (2009).

[26] S. Jakiela, P. M. Korczyk, S. Makulska, O. Cybulski, and P. Garstecki, Phys. Rev. Lett. 108, 134501 (2012).

[27] J. D. Tice, H. Song, A. D. Lyon, and R. F. Ismagilov, Langmuir 19, 9127 (2003).

[28] P. Garstecki, M. J. Fuerstman, H. A. Stone, and G. M. Whitesides, Lab. Chip 6, 437 (2006).

[29] S. L. Anna, N. Bontoux, and H. A. Stone, Appl. Phys. Lett. 82, 364 (2003).

[30] H. Song and R. F. Ismagilov, J. Am. Chem. Soc. 125, 14613 (2003).

[31] A. D. Griffiths and D. S. Tawfik, Trends Biotechnol. 24, 395 (2006).

[32] B. T. Kelly, J. C. Baret, V. Taly, and A. D. Griffiths, Chem. Commun. 18, 1773 (2007).

[33] P. Panizza, W. Engl, C. Hany, and R. Backov, Colloids Surf. A 312, 24 (2008).

[34] O. Cybulski and P. Garstecki, Phys. Rev. E, 82, 056301 (2010).

[35] J. C. McDonald, D. C. Duffy, J. R. Anderson, D. T. Chiu, H. Wu, O .J. A. Schueller, and G. M. Whitesides, Electrophoresis 21, 27 (2000).

[36] D. R. Link, S. L. Anna, D. A. Weitz, and H. A. Stone, Phys. Rev. Lett. 92, 054503 (2004).

[37] L. Ménétrier-Deremble and P. Tabeling, Phys. Rev. E 74, 035303(R) (2006).

[38] M.-C. Jullien, M.-J. Tsang Mui Ching, C. Cohen, L. Menetrier, and P. Tabeling, Phys. Fluids 21, 072001 (2009).

[39] L. Salkin, L. Courbin, and P. Panizza, Phys. Rev. E 86, 036317 (2012).

[40] L. Salkin, A. Schmit, L. Courbin, and P. Panizza, Lab. Chip 13, 3022 (2013). 